\title{
Colour flow Doppler mapping in the assessment of prosthetic valve regurgitation
}

\author{
JOHN CHAMBERS MARK MONAGHAN, GRAHAM JACKSON \\ From the Cardiac Department, King's College Hospital, London
}

SUMmaRY Two hundred Carpentier-Edwards, Björk-Shiley, and Starr-Edwards prostheses in 173 patients were examined. Sixteen $(16 \%)$ in the aortic and $24(25 \%)$ in the mitral position were associated with clinical signs of regurgitation. A phased array system (Hewlett-Packard A77020A) with a $2.5 \mathrm{MHz}$ duplex and $1.9 \mathrm{MHz}$ continuous wave transducer was used. Colour flow mapping showed trivial transvalvar regurgitation in $23(53 \%)$ metal aortic prostheses, and only nine $(20 \%)$ metal mitral prostheses. This difference was probably attributable to shielding of the left atrium by the metal components. Colour mapping confirmed abnormal regurgitation in all aortic prostheses with early diastolic murmurs, but regurgitation was also shown in $25(29 \%)$ with no diastolic murmur. Abnormal mitral regurgitation was found in $13(54 \%)$ patients with a pansystolic murmur, but also in six $(8 \%)$ with no systolic murmur. Two patients, thought on clinical grounds to have mild mitral regurgitation, had unexpectedly large jets on colour flow mapping. About one in three prostheses had paraprosthetic leaks, $65(79 \%)$ of which were small with a jet area less than $20 \%$ of the area of the receiving chamber. The development of new paraprosthetic leaks led to the diagnosis of bacterial endocarditis in two patients. In eight patients regurgitation was first diagnosed with continuous wave Doppler, but was afterwards shown with colour mapping and in a further 10 regurgitation could only be shown by continuous wave Doppler.

Colour flow mapping was less sensitive than continuous wave Doppler in detecting regurgitation, but seemed able to distinguish normal transvalvar from paraprosthetic regurgitation. Further studies in the natural course of paraprosthetic leaks and a comparison of the transoesophageal and transthoracic approaches in the assessment of mitral prostheses are needed.

It is important to be able to detect and localise the site of regurgitation associated with prosthetic valves. The development of a new leak in a paraprosthetic position may suggest bacterial endocarditis or in a transvalvar position may be the first indication of primary failure in a bioprosthesis. It is also useful to be able to guide the surgeon when repair of a paraprosthetic leak is required.

Continuous wave Doppler ultrasound is highly sensitive for the detection of regurgitation, but it cannot pinpoint the leak. Although localisation is possible with conventional pulsed Doppler, it is time consuming and of limited accuracy. ${ }^{1-3}$ Colour flow Doppler mapping gives a cross sectional version of conventional pulsed Doppler. Velocity and directional information are collected at multiple depths

Requests for reprints to Dr John Chambers, Cardiac Unit, Guy's Hospital, St Thomas Street, London SE1 9RT.

Accepted for publication 24 January 1989 down each scan line and are then superimposed on a cross sectional echocardiographic image in real time. Colour flow Doppler mapping is highly sensitive for detecting regurgitation in native valves, ${ }^{45}$ but there are no published studies of its application in the assessment of regurgitation associated with prosthetic valves.

The aim of this study was to establish the role and likely benefits of colour flow Doppler mapping in assessing the incidence, severity, and localisation of regurgitant jets in prosthetic valves.

\section{Patients and methods}

PATIENTS

Consecutive patients with prosthetic valves referred either for routine echocardiography or with suspected prosthetic regurgitation were studied from 1 December 1986 to 30 March 1988. Patients with tricuspid valve prostheses or aortic and mitral prostheses other than Carpentier-Edwards, Björk- 


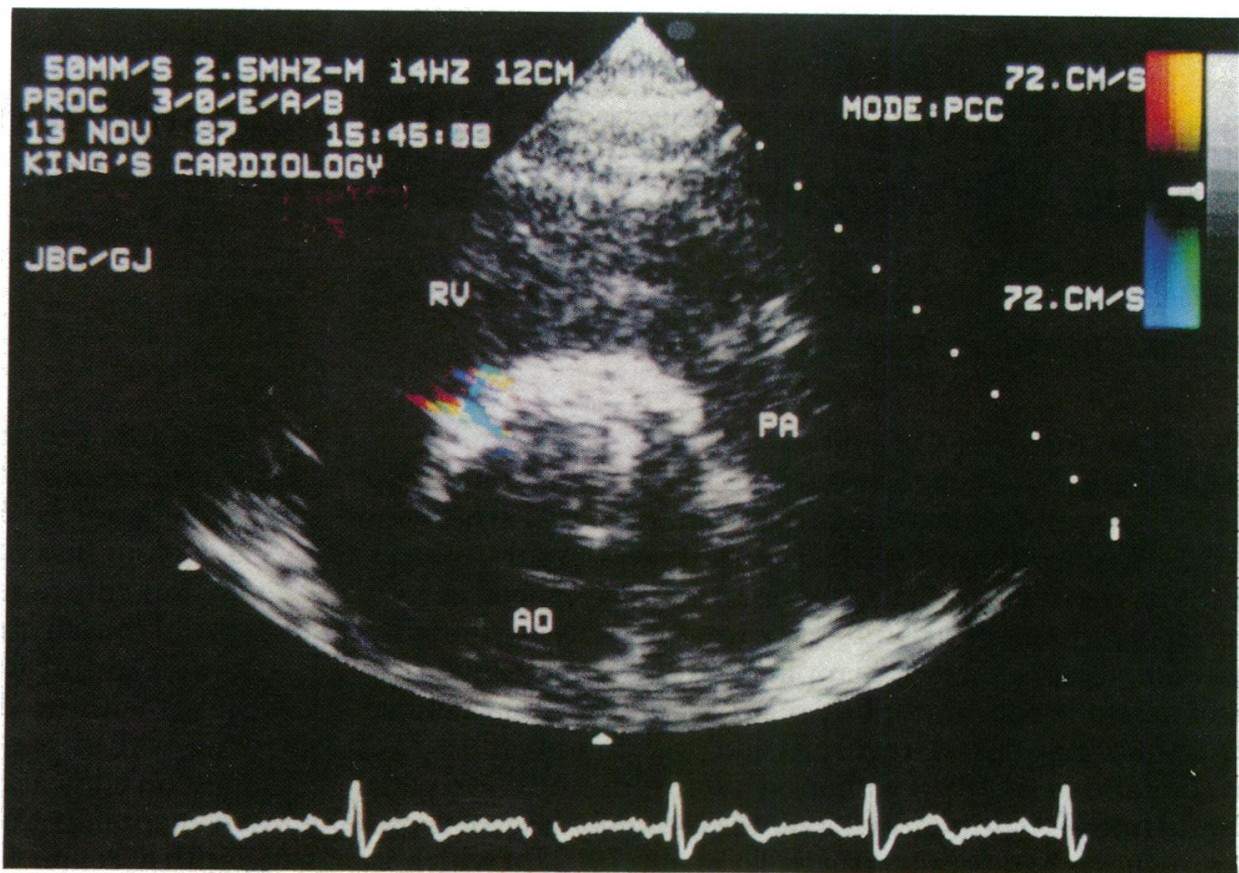

Fig 1 Paraprosthetic aortic regurgitation. Parasternal short axis view of a Starr-Edwards aortic prosthesis. The base of the jet was clearly seen in the anterior part of the sewing ring at about 11 o'clock.

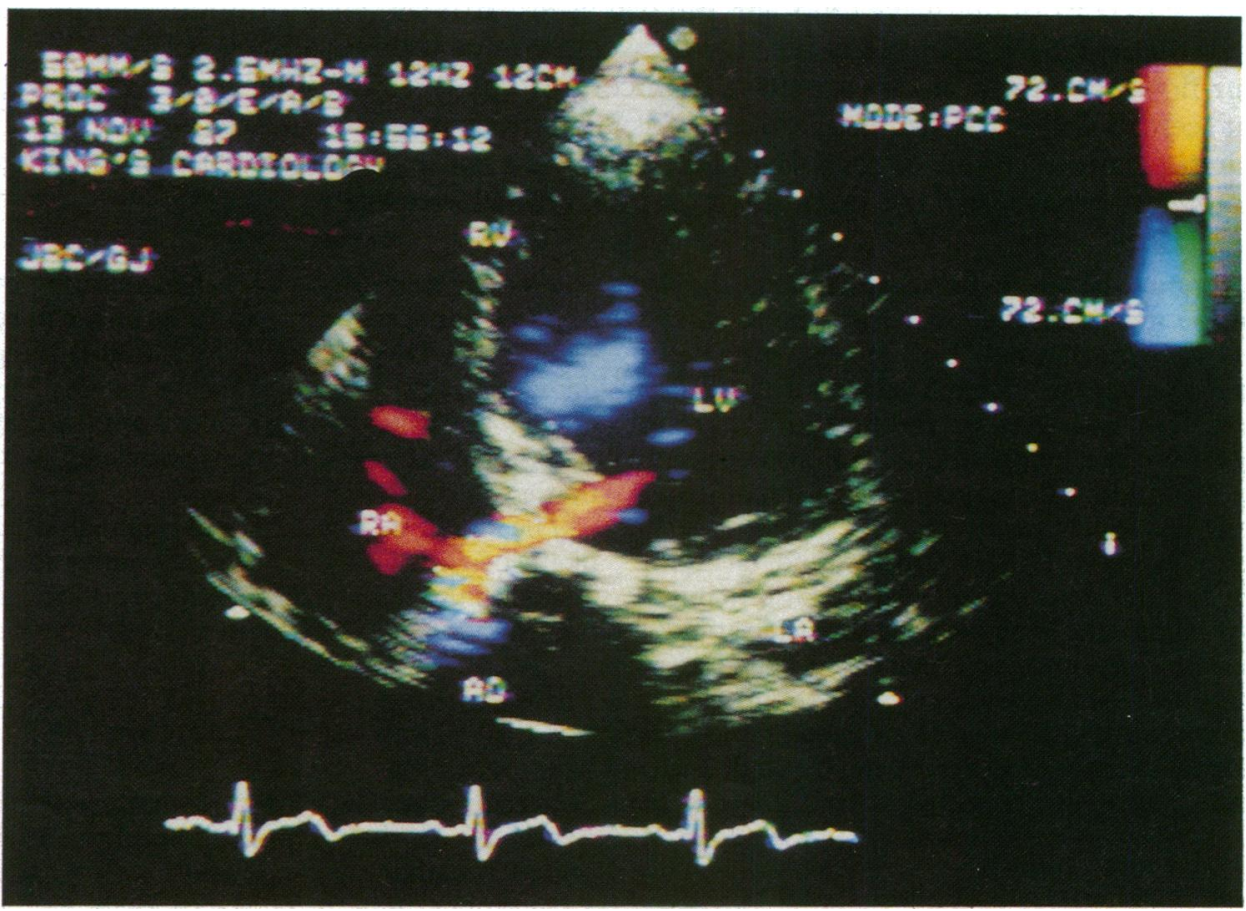

Fig 2 Paraprosthetic aortic regurgitation. Apical five chamber view showing the Starr-Edwards aortic prosthesis in fig 1. A stream of colour originated in the aartic root and was seen passing around the sewing ring and into the left ventricle. 


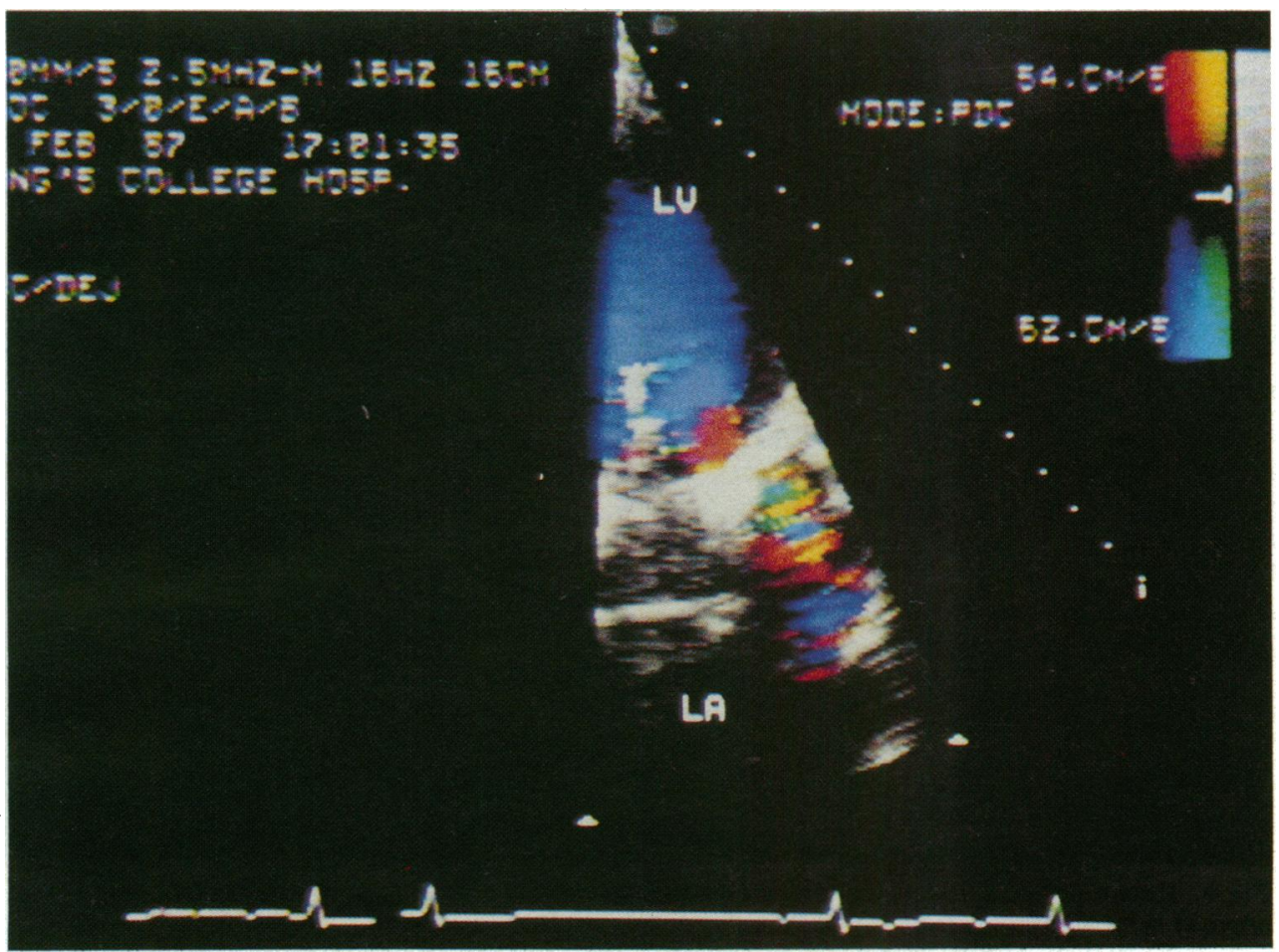

Fig 3 Paraprosthetic aortic regurgitation. Apical four chamber view of a Starr-Edwards mitral valve. Flow acceleration (red) was seen within the left ventricle at the edge of the sewing ring. There was a mitral regurgitant jet in the left atrium, but no clear stream of colour around the prosthesis.

Shiley, or Starr-Edwards were excluded because there were too few of them. This left 200 prostheses in 173 patients. There were 103 aortic prostheses in which $16(16 \%)$ had the murmur of aortic regurgitation and 97 mitral prostheses of which $24(25 \%)$ had pansystolic murmurs.

\section{APPARATUS}

We used a phased array ultrasound system (HewlettPackard A77020A) with a $1.9 \mathrm{MHz}$ continuous wave Doppler probe and a duplex $2.5 \mathrm{MHz}$ transducer for imaging and colour flow Doppler mapping. The colour flow Doppler system was set to maximum packet size and minimum reject with the gain just below the level at which ambiguous colour flooding occurred. Both enhanced and variance threshold maps were used. Recordings were made on to professional quality three quarter inch $\mathrm{U}$-matic tape.

\section{STUDY}

A clinical examination was performed to look for evidence of prosthetic dysfunction or coexistent tricuspid regurgitation. Cross sectional imaging with colour flow Doppler was then performed in all conventional approaches and in addition the trans- ducer was tilted in an attempt to detect areas of abnormal flow in more anterior and posterior positions. The parasternal short axis view was found to be particularly useful for localising aortic jets (fig 1). A continuous wave examination was then performed from the apical approach with multiple gain settings and angulations. If a regurgitant jet was shown for the first time on continuous wave Doppler a second attempt was made to detect it by colour flow Doppler mapping.

Regurgitation was considered to be paraprosthetic if: (a) a stream of colour was visible around the sewing ring in mitral prostheses during systole and in aortic prostheses during diastole (fig 2 ); (b) the origin of the regurgitant jet was visible at a point outside the sewing ring although there was no visualisation of flow around the valve (fig 3); (c) the origin of the regurgitant jet was in the region of the sewing ring and although not clearly outside the ring, was separate from forward flow (fig 4).

Regurgitation was considered to be transvalvar if: (a) its origin was seen within the orifice (as defined by the forward flow pattern) in at least two different views (fig 5); (b) in metal mitral prostheses systolic retrograde flow was seen originating at the edge of the 


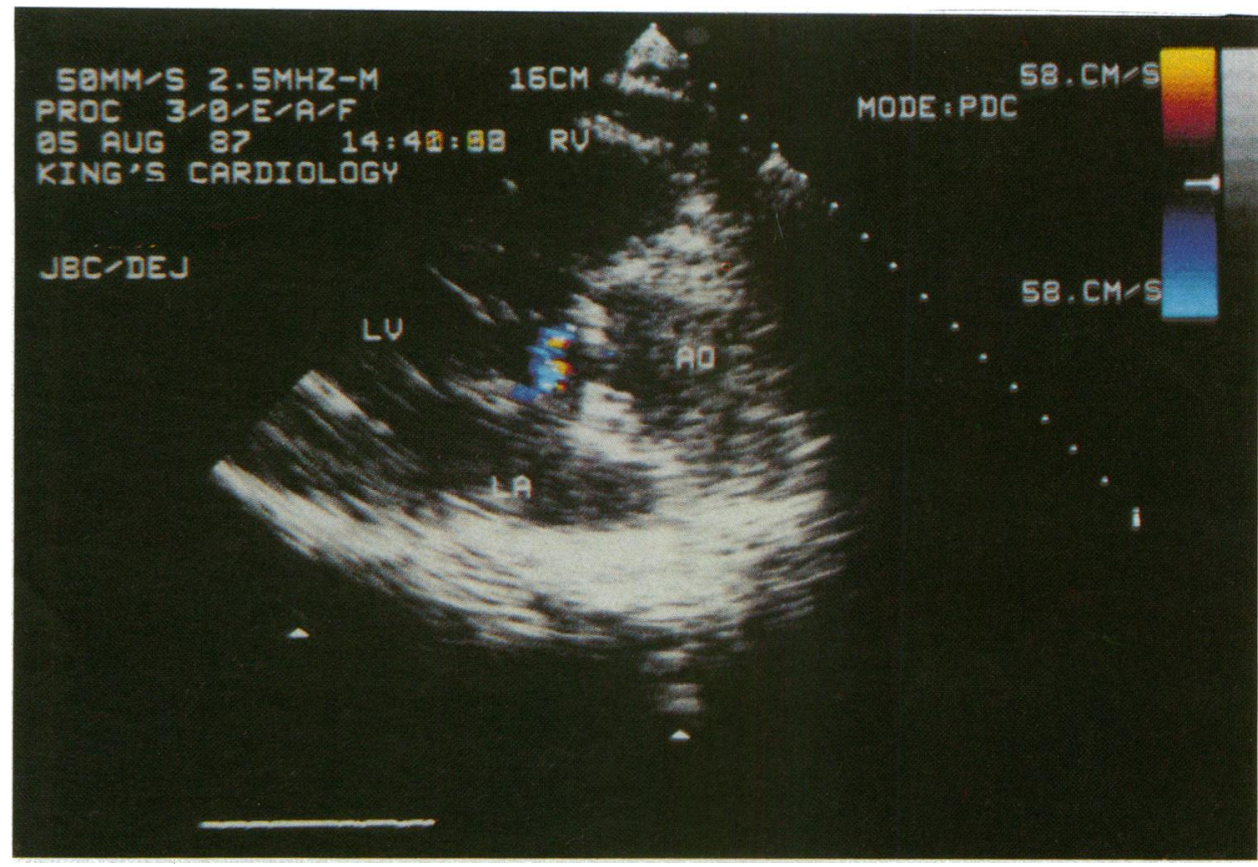

Fig 4 Paraprosthetic aortic regurgitation. Parasternal long axis view of a Björk-Shiley prosthesis. The origin of the jet is separate from forward flow.

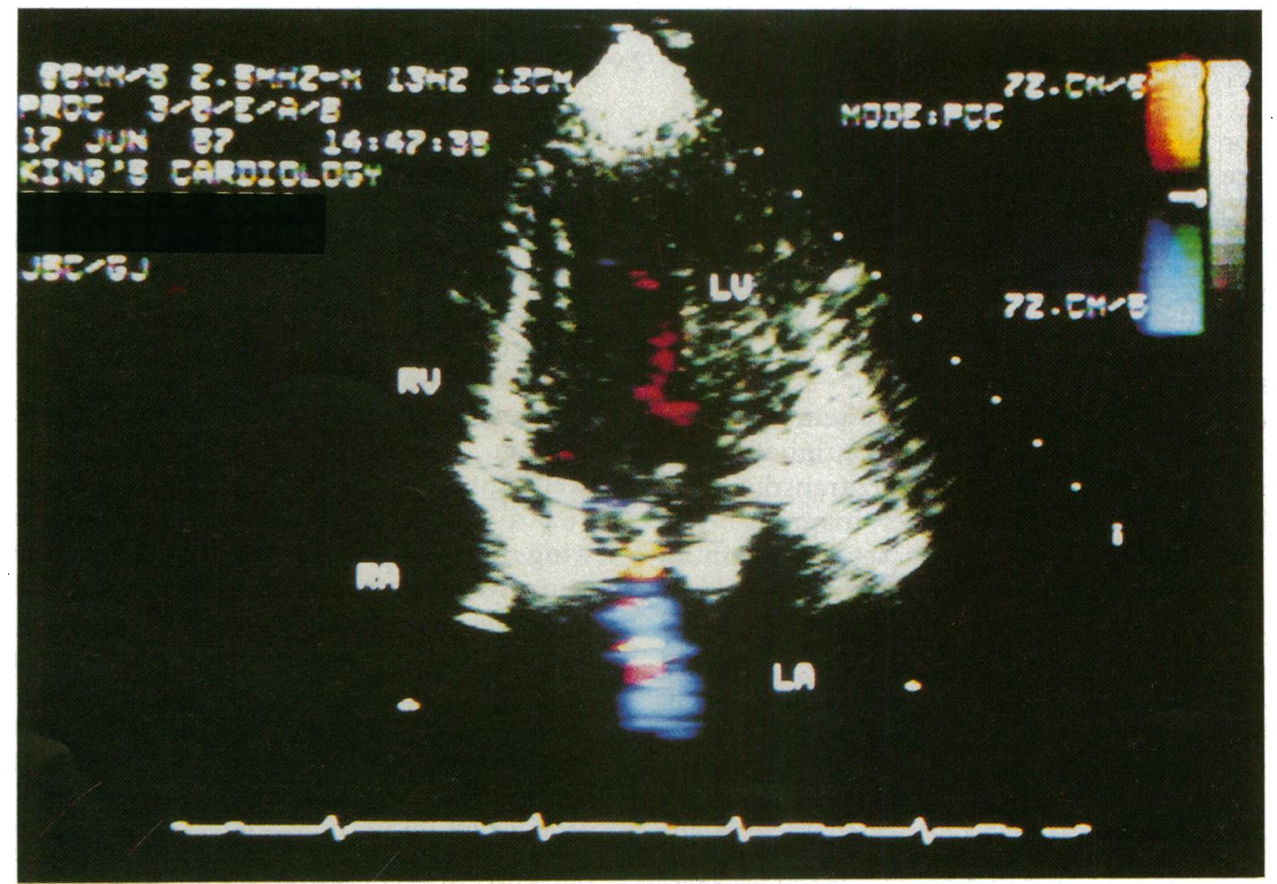

Fig 5 Regurgitation through a mitral prosthesis. An apical four chamber view of a Carpentier-Edwards prosthesis. The jet also appeared inside the valve ring in the parasternal long axis view. 


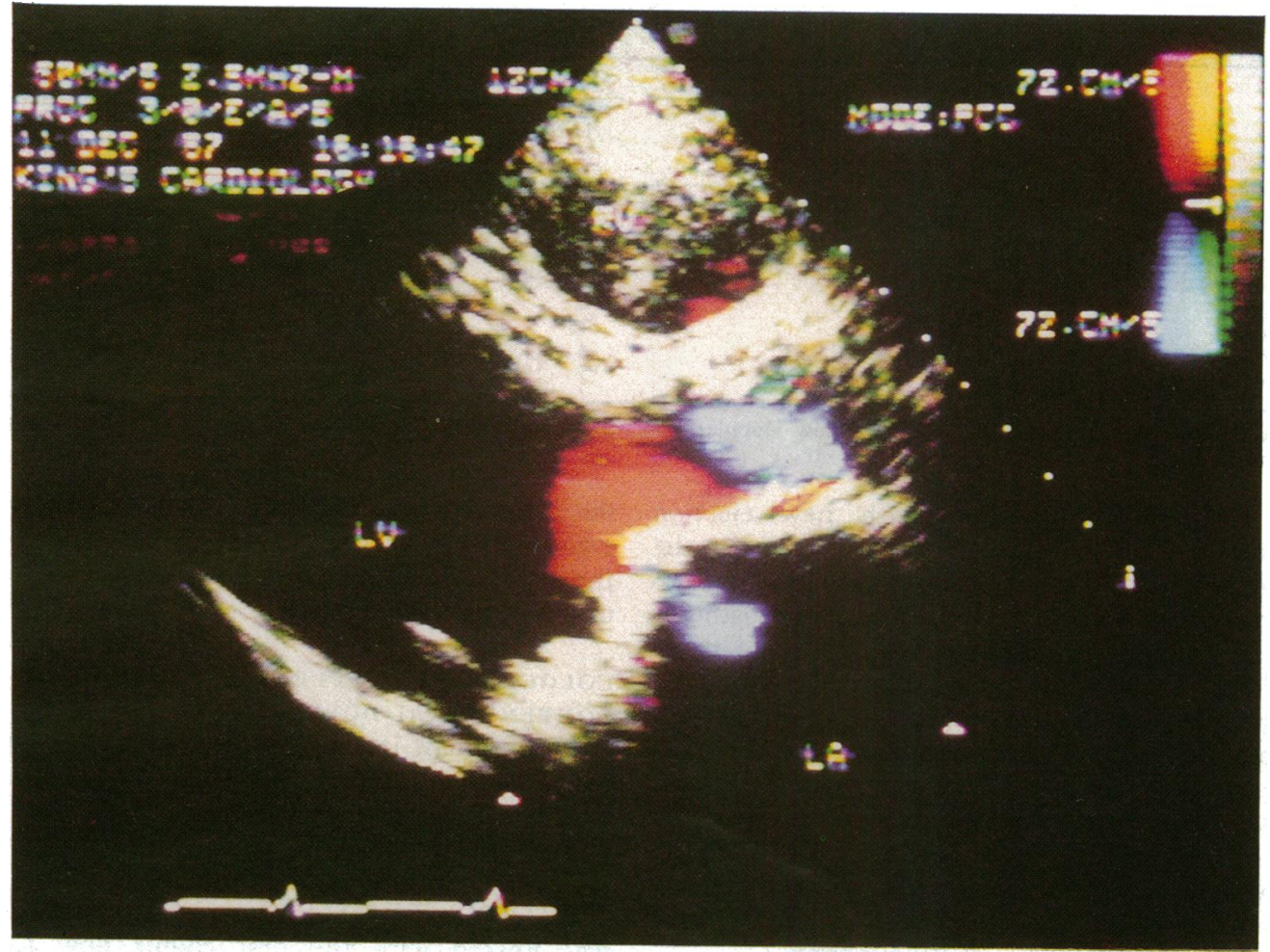

Fig 6 Parasternal long axis view of transvalvar regurgitation. A high velocity jet was recorded by continuous wave. On colour flow Doppler mapping, there was high velocity flow (blue) between the disc and the sewing ring continuous with a billowing low velocity jet seen within the left atrium in systole.

occluder (disk or ball) in the parasternal long axis view (fig 6); or (c) beyond the shielding of the occluder in the absence of evidence of a paraprosthetic leak.

The severity of paraprosthetic aortic regurgitation was estimated from the arc occupied by the base of the jet around the sewing ring. The maximum area of the aortic or paraprosthetic mitral jet was also expressed as a percentage of the receiving chamber area ${ }^{6}$ when the approach that gave optimal imaging of the jet was used. A semiquantitative estimate of jet area in transmitral regurgitation was not attempted because of shielding by the metal components.

Five patients with severe prosthetic dysfunction were evaluated by cardiac catheterisation and contrast injection-four proceeded to operation. One further patient had an operation without previous catheterisation and four cases had catheterisation for coronary artery disease and the prosthesis was also evaluated at this time.

\section{Results}

INCIDENCE OF REGURGITATION

Regurgitation was detected in $97(49 \%)$ prostheses (table 1). Trivial transvalvar regurgitation was shown more commonly in the Björk-Shiley (63\%) than the Starr-Edwards $(27 \%)$ prostheses in the aortic position, but in similar frequencies in the mitral position. Paraprosthetic leaks occurred in 73\% Starr-Edwards aortic prostheses, in 6\% Carpentier-Edwards mitral prostheses, and in $21-28 \%$ of Starr-Edwards mitral, Carpentier-Edwards aortic, Björk-Shiley mitral, and Björk-Shiley aortic. Of the 12 Carpentier-Edwards prostheses with transvalvar regurgitation, all but one had been in position for longer than 48 months (mean 77 (29) months). Of those with paraprosthetic regurgitation, eight $(40 \%)$ had been in position for less than 12 months. It was impossible to localise the site of regurgitation in one Carpentier-Edwards prosthesis where the base of the jet was broad.

Aortic regurgitation was confirmed in all prostheses with an early diastolic murmur, but mild paraprosthetic regurgitation was also found in a further $25(29 \%)$ of 87 patients with no murmur. Of the 24 cases with pansystolic murmurs, $13(54 \%)$ had mitral regurgitation and in the others only tricuspid regurgitation was found. One mitral prosthesis with a high pitched ejection systolic murmur had minor regurgitation originating at the base of one cusp. 
Table 1 Occurrence of regurgitation shown by ultrasound (percentages in parentheses)

\begin{tabular}{|c|c|c|c|c|c|c|c|c|}
\hline \multirow[b]{3}{*}{$\begin{array}{l}\text { Total frequency } \\
\text { PP on CFD } \\
\text { Transvalvar on CFD } \\
\text { PP and transvalvar } \\
\text { Uncertain } \\
\text { Multiple PP } \\
\text { On CW not CFD } \\
\text { Murmur }\end{array}$} & \multicolumn{2}{|c|}{ Carpentier-Edwards } & \multicolumn{2}{|c|}{ Björk-Shiley } & \multicolumn{4}{|c|}{ Starr-Edwards } \\
\hline & $\begin{array}{l}A V R \\
(n=60)\end{array}$ & $\begin{array}{l}M V R \\
(n=53)\end{array}$ & $\begin{array}{l}A V R \\
(n=32)\end{array}$ & $\begin{array}{l}M V R \\
(n=29)\end{array}$ & \multicolumn{2}{|c|}{$\begin{array}{l}A V R \\
(n=11)\end{array}$} & \multicolumn{2}{|c|}{$\begin{array}{l}M V R \\
(n=15)\end{array}$} \\
\hline & $\begin{array}{rr}23 & (38) \\
17 & (28) \\
6 & (10) \\
1 & (2) \\
1 & \\
1 & \\
0 & \\
8 & (13)\end{array}$ & $\begin{array}{rr}11 & (21) \\
3 & (6) \\
6 & (11) \\
0 & \\
0 & \\
& \\
2 & \\
7 & (13)\end{array}$ & $\begin{array}{rr}30 & (94) \\
9 & (28) \\
20 & (63) \\
3 & (9) \\
0 & \\
1 & \\
4 & (13) \\
5 & (16)\end{array}$ & $\begin{aligned} 17 & (59) \\
6 & (21) \\
7 & (24) \\
0 & \\
0 & \\
1 & \\
4 & (14) \\
4 & (14)\end{aligned}$ & $\begin{array}{r}10 \\
8 \\
3 \\
2 \\
0 \\
0 \\
1 \\
3\end{array}$ & $\begin{array}{l}(91) \\
(73) \\
(27) \\
(18)\end{array}$ & $\begin{array}{l}6 \\
4 \\
2 \\
0 \\
0 \\
0 \\
0 \\
2\end{array}$ & $\begin{array}{l}(40) \\
(27) \\
(13)\end{array}$ \\
\hline
\end{tabular}

AVR, aortic valve replacement; CFD, colour flow Doppler; CW, continuous wave Doppler; MVR, mitral valve replacement; PP, paraprosthetic.

Two further mitral valves with new ejection murmurs had demonstrable flow acceleration in the left ventricular outflow tract thought to be related to the tip of one of the stents. Of the 73 mitral prostheses with no pansystolic murmur, six $(8 \%)$ had paraprosthetic mitral regurgitation. Most cases of severe prosthetic dysfunction were suspected clinically, but in two severe paraprosthetic mitral regurgitation was shown by colour flow Doppler despite only soft pansystolic murmurs. In two patients the development of new paraprosthetic regurgitation led to the diagnosis of bacterial endocarditis. A further 11 patients with unexplained breathlessness were shown to have normal prosthetic function.

SEVERITY OF REGURGITATION (TABLE 2)

Most of the regurgitant jets were small. The area of the jet in $65(79 \%$ ) was less than $20 \%$ of the receiving chamber area. Shielding by the prosthesis precluded reliable estimation of the severity of regurgitation through metal mitral prostheses. Paraprosthetic mitral regurgitant areas were also likely to have been underestimated because of partial shielding of the jet.

The base of the aortic jet involved approximately

Table 2 Severity of regurgitation

\begin{tabular}{|c|c|c|c|c|}
\hline & $<10 \%$ & $10-19 \%$ & $20-40 \%$ & $>40 \%$ \\
\hline \multicolumn{5}{|l|}{ Aortic CE: } \\
\hline $\begin{array}{l}\text { PP } \\
\text { Through }\end{array}$ & 4 & 11 & 2 & 0 \\
\hline $\begin{array}{l}\text { Through } \\
\text { Mitral CE: }\end{array}$ & 1 & 3 & 1 & 1 \\
\hline $\begin{array}{l}\text { Mitral CE: } \\
\text { PP }\end{array}$ & 2 & 1 & 0 & \\
\hline Through & 4 & 0 & 1 & $\begin{array}{l}0 \\
1\end{array}$ \\
\hline \multicolumn{5}{|l|}{$\begin{array}{l}\text { Through } \\
\text { Aortic BS: }\end{array}$} \\
\hline PP & 2 & 2 & 3 & 2 \\
\hline $\begin{array}{l}\text { Through } \\
\text { Mitral BS: }\end{array}$ & 13 & 7 & 0 & 0 \\
\hline $\begin{array}{l}\text { Through } \\
\text { Mitral BS: }\end{array}$ & 2 & 2 & 2 & 0 \\
\hline \multicolumn{5}{|l|}{ Aortic SE: } \\
\hline $\begin{array}{l}\text { PP } \\
\text { Through }\end{array}$ & 2 & 4 & 1 & 1 \\
\hline \multicolumn{5}{|l|}{$\begin{array}{l}\text { Through } \\
\text { Mitral SE: }\end{array}$} \\
\hline PP & 1 & 1 & 2 & 0 \\
\hline
\end{tabular}

CE, Carpentier-Edwards; BS, Björk-Shiley; PP, paraprosthetic; SE, Starr-Edwards. $90^{\circ}$ of the ring in one prosthesis, approximately $45^{\circ}$ in eight, and $<20^{\circ}$ in the remaining 37 . Table 3 shows the distribution of the jets. The jets were multiple in origin in three prostheses.

\section{COLOUR FLOW DOPPLER COMPARED WITH CONVENTIONAL DOPPLER}

All regurgitant jets shown by colour flow Doppler were also recorded by continuous wave or conventional pulsed Doppler. There were, however, 10 patients in whom low intensity regurgitant jets shown by continuous wave were not seen on colour flow Doppler. In eight further patients, regurgitation was missed with the first colour flow Doppler examination but shown at a subsequent examination.

There were 15 patients with regurgitant prostheses who had been examined by conventional Doppler ultrasound alone and in whom it had not been possible confidently to localise the jet. We thought that localisation was possible with colour flow Doppler in all of these patients.

\section{COMPARISON WITH CATHETER OR FINDINGS AT OPERATION}

Cardiac catheterisation was performed in five patients and confirmed the Doppler findings in all of them. There was mild regurgitation through a severely stenotic prosthesis in two patients and

Table 3 Localisation of paraprosthetic regurgitant jets

\begin{tabular}{lllll}
\hline Type of valve & Anterior & Posterior & $R V$ side & LV side \\
\hline Aortic: & & & & \\
CE & 8 & 7 & 5 & 1 \\
BS & 6 & 1 & 2 & 1 \\
SE & 3 & 3 & 1 & 1 \\
& & & & \\
Mitral: & Anterior & Posterior & Septal & Free wall \\
CE & 1 & 1 & 1 & 0 \\
BS & 4 & 0 & 1 & 2 \\
SE & 1 & 0 & 1 & 2
\end{tabular}

CE, Carpentier-Edwards; BS, Björk-Shiley; LV, left ventricle; RV, right ventricle; SE, Starr-Edwards. 
severe paraprosthetic regurgitation in three. At operation, however, one patient who was thought to have a paraprosthetic leak was found to have a sinus between the base of the cusp and the stent, but no dehiscence of the sewing ring. The colour flow findings were confirmed in the other four patients. Two patients thought to have considerable paraprosthetic regurgitation had severe intercurrent illness which prevented further intervention. Catheterisation was also performed for suspected coronary artery disease in four patients with CarpentierEdwards prostheses; it confirmed the absence of regurgitation.

\section{Discussion}

This is the first study to use colour flow Doppler mapping to describe patterns of regurgitation in a consecutive series of patients with prosthetic valves. The striking finding was the high frequency of unsuspected paraprosthetic regurgitation in prostheses of all three types in the aortic position and in Björk-Shiley and Starr-Edwards prostheses in the mitral position.

Most of the regurgitant jets were, however, small and probably not haemodynamically important. Furthermore, most severely dysfunctioning prostheses were diagnosed clinically. Was colour flow mapping, therefore, of clinical use? Two patients with severe paraprosthetic regurgitation were first diagnosed with colour flow Doppler and in two further patients newly developed mild regurgitation led to the diagnosis of bacterial endocarditis. Localisation of the site of regurgitation guided the surgeon in all three patients with paraprosthetic leaks who underwent reoperation. In all 24 mitral prostheses where a new systolic murmur was heard after operation, colour flow mapping was able to localise the origin of the flow disturbance to the mitral or tricuspid valve or to the left ventricular outflow tract. It may be difficult clinically to exclude mitral prosthetic regurgitation in a patient with coexistent tricuspid regurgitation. Colour flow mapping also contributed by excluding significant prosthetic regurgitation in 11 patients in whom the cause of failure to improve after operation was not obvious clinically.

Paraprosthetic leaks may occur because the bed created by scraping away calcified tissue is never perfectly circular so that the prosthesis cannot always fit snugly. Regurgitation may be less common in the Carpentier-Edwards mitral prostheses because the annulus is covered in sponge and may allow a better fit than the rings of metal prostheses. The state of the recipient tissue, the type and technique of suturing, and the methods of myocardial preservation may all contribute to the development of paraprosthetic regurgitation and future studies should evaluate these prospectively.

Normally functioning Björk-Shiley and StarrEdwards prostheses have trivial transvalvar leaks during closure. In the aortic prostheses these jets were always small, less than $20 \%$ of the receiving chamber area, and usually narrow. Twin jets were sometimes seen. Transvalvar regurgitation was seen less commonly in the mitral prostheses. In this position, access can be improved by the use of the transoesophageal approach, which allows interrogation of the left atrium without the problem of shielding. It is likely that shielding by mitral prostheses was the reason why paraprosthetic regurgitation was seen more frequently with valves in the aortic rather than the mitral position. There is, however, no published study describing the results that can be obtained with the transoesophageal approach and how often clinically important information gained by this route is missed by the transthoracic approach. Most studies with conventional Doppler reported lower incidences of regurgitation than shown here, but they were studies of small numbers. ${ }^{278} \mathrm{~A}$ study of the functioning of BjörkShiley prostheses by colour flow Doppler failed to find regurgitation in any of the 10 aortic prostheses examined. ${ }^{9}$ The fact that we detected regurgitation in $63 \%$ of aortic prostheses probably reflects the increased sensitivity of the apparatus that we used.

There is uncertainty about whether any regurgitation through Carpentier-Edwards prostheses can be regarded as normal. Conventional ultrasound showed regurgitation in $8-26 \%$ of aortic prostheses ${ }^{81011}$ and in 4-11\% of mitral prostheses. ${ }^{1011} \mathrm{We}$ found valvar regurgitation in $10 \%$ of the aortic and $11 \%$ of the mitral valves. Only one regurgitant prosthesis had been in position for less than 48 months; this suggests that transvalvar regurgitation is a sign of primary failure.

Systematic validation with angiography of the localisation of regurgitation by colour Doppler mapping is not a practical proposition. Furthermore, angiography may be less accurate than Doppler ultrasound $^{3}$ and it is possible that colour flow Doppler mapping will soon be considered the reference standard for localisation. In one case in this series a leak at the base of the cusp was wrongly diagnosed as being paraprosthetic. This emphasises that colour flow Doppler mapping may not always reliably distinguish between eccentric jets through a prosthesis and paraprosthetic leaks. When abnormal flow is clearly seen around the sewing ring it is unlikely that mistakes can be made.

The area of the regurgitant jet shown by colour flow Doppler is dependent on gain and instrument 
settings ${ }^{12} 13$ and may not always give an accurate assessment of the severity of regurgitation. Helmcke et al, however, have shown a good correlation between the angiographically assessed severity and mitral regurgitant jet area expressed as a percentage of left atrial area. ${ }^{14}$ Most patients with mild regurgitation by angiographic criteria had regurgitant areas of less than $20 \%$ and most with severe regurgitation had areas over $40 \%$.

We conclude that colour flow Doppler mapping detects most expected and abnormal regurgitant jets, most of which are small and probably not haemodynamically important. Occasionally, however, they are more severe than had been suspected on clinical grounds. New paraprosthetic leaks suggest the possibility of bacterial endocarditis. The technique is useful for the localisation of new murmurs appearing after mitral valve replacement and probably for excluding important valve dysfunction. Further studies of the natural course of paraprosthetic regurgitation and comparative studies of the transoesophageal and transthoracic approaches in the assessment of prostheses should now be undertaken.

JC holds a British Heart Foundation Fellowship. Mr Kerry Packer donated the colour flow system.

\section{References}

1 Veyrat C, Wichitz S, Lessana A, Ameur A, Abitbol G, Kalmanson D. Valvar prosthetic dysfunction. Localisation and evaluation of the dysfunction using the Doppler technique. Br Heart J 1985;54:273-84.

2 Sagar KB, Wann S, Paulsen WHJ, Romhilt DW. Doppler echocardiographic evaluation of Hancock and Björk-Shiley prosthetic valves. J Am Coll Cardiol
1986;7:681-7.

3 Chambers JB, Monaghan MJ, Jackson G, Jewitt DE. Doppler echocardiographic appearance of cusp tears in tissue valve prostheses. J Am Coll Cardiol 1987; 10:462-6.

4 Switzer DF, Nanda NC. Doppler color flow mapping. Ultrasound Med Biol 1985;11:403-16.

5 Omoto R, Yokote Y, Takamoto S, et al. The development of real-time two-dimensional Doppler echocardiography and its clinical significance in acquired valvular diseases. Jpn Heart $J$ 1984;25:325-40.

6 Miyatake H, Izumi S, Okamoto M, et al. Semiquantitative grading of severity of mitral regurgitation by real-time, two-dimensional Doppler flow imaging technique. J Am Coll Cardiol 1986;7:82-8.

7 Panidis IP, Ross J, Mintz GS. Normal and abnormal prosthetic valve function as assessed by Doppler echocardiography. J Am Coll Cardiol 1986;8:317-26.

8 Ramirez ML, Wong M, Sadler N, Shah PM. Doppler evaluation of bioprosthetic and mechanical aortic valves: data from four models in 107 stable ambulatory patients. Am Heart J 1988;115:418-25.

9 Dittrich H, Nicod P, Hoit B, Dalton N, Sahn D. Evaluation of Björk-Shiley prosthetic valves by realtime two-dimensional Doppler echocardiographic flow mapping. Am Heart J 1988;115:133-8.

10 Cooper DM, Stewart WJ, Schiavone WA, et al. Evaluation of normal prosthetic valve function by Doppler echocardiography. Am Heart J 1987;114:576-82.

11 Gibbs JL, Wharton GA, Williams GJ. Doppler echocardiographic characteristics of the CarpentierEdwards xenograft. Eur Heart J 1986;7:353-6.

12 Sahn DJ, Chung KJ, Tamura T, et al. Factors affecting jet visualisation by color flow mapping Doppler echo: in vitro studies [Abstract]. Circulation 1986;74(suppl II):271.

13 Otsuji Y, Tei C, Kisanuki A, Natsugoe K, Kawazoe Y. Color Doppler echocardiographic assessment of the change in the mitral regurgitant volume. Am Heart $J$ 1987;114:349-54.

14 Helmcke F, Nanda NC, Hsuing MC, et al. Color Doppler assessment of mitral regurgitation with orthogonal planes. Circulation 1987;75:175-83. 